\title{
NHÂ̂N XÉT GIÁ TRỊ HỖ TRỢ CHẨN ĐOÁN BỆNH RĂNG KHÔN MỌC LỆCH CỦA HỌC MÁY
}

\section{TÓM TẮT}

Mục tiêu nghiên cứu: Nghiên cứu được thực hiện trên 100 phim XQ của bệnh nhân có răng khổn mọc lệch để xác định khả năng hỗ trợ chẩn đoán của phương pháp hoc máy. Đối tượng và phương pháp nghiên cứu: Phần mềm học máy được xây dựng trên tâp dữ liêu có 504 phim XQ Panorama, nghiên cứu thử nghiệm được thiết kế theo phương pháp thử nghiệm lâm sàng để xác định đô nhạy và độ đăc hiệu của phần mềm học máy. Kểt quả: Trong tổng số 187 răng khôn hàm dưới, theo bác sỹ chẩn đoán 63 răng mọc thẳng, chiếm 33,7\%, 124 răng mọc lệch, chiếm $66,3 \%$. Theo phân loại, loại 1 có 42 răng $(22,5 \%)$, loại 2 có 81 răng $(43,3 \%)$ và loại 4 có 1 răng $(0,5 \%)$. Khi sử dụng phần mềm để chẩn đoán: máy chân đoán được 187 răng (100\%). Máy chẩn đoán giống bác sỹ ở 149 răng (79,68\%). Kết luân: Khi sử dụng học máy để hố trợ chẩn đoán bệnh lý răng khôn mọc lệch: độ nhạy, độ đặc hiệu tương ứng lần lượt là 98,5\%; $86 \%$ khi chẩn đoán có hay không có bệnh lý.

Từ khoá: chẩn đoán, răng khôn, học máy...

\section{SUMMARY \\ EVALUATING THE VALUE OF DIAGNOSIS SUPPORT OF MACHINE LEARNING IN DIAGNOSIS OF MALPOSITION WISDOM TOOTH}

Research objectives: The study was conducted on $100 \mathrm{X}$-ray films of patients with malpositon wisdom teeth to determine the capability of the diagnostic support of machine learning methods. Research subjects and methods: Machine learning software was built on the data set with 504 dental panoramic radiographs, the clinical trial was designed according to determining the sensitivity and specificity of the software. Results: In the total amount of 187 lower third molars, the dentist diagnosed 63 normal teeth, accounting for $33.7 \%, 124$ malposition teeth, accounting for $66.3 \%$. According to classification, type 1 has 42 teeth (22.5\%), type 2 has 81 teeth (43.3\%) and type 4 has 1 tooth $(0.5 \%)$. When using software to diagnose: the learning machine could diagnose 187 teeth $(100 \%)$. The learning machine had the same diagnosis with doctor in 149 teeth (79.68\%). Conclusion: When using learning machine to support the diagnosis of malposition wisdom tooth pathology: the sensitivity and specificity were respectively $98.5 \%$; $86 \%$ at the diagnosis with or without pathology.

${ }^{1}$ Viện Đào tạo Răng Hàm Mặt-Trường Đại Học Y Hà Nội ${ }^{2}$ Bệnh viện Viêt Nam Cuba Hà Nội

Chiu trách nhiệm chính: Võ Trương Như Ngọc

Email: votruongnhungoc@gmail.com

Ngày nhận bài: 12.3.2021

Ngày phản biện khoa học: 10.5.2021

Ngày duyệt bài: 18.5.2021
Võ Trương Như Ngọc ${ }^{1}$, Phùng Thị Thu Hà ${ }^{2}$

Key words: diagnostic, wisdom teeth, deep learning...

\section{I. ĐẶT VẤN ĐỀ}

Hiện nay, tại các bệnh viện hay phòng khám nha khoa tư nhân, các bác sĩ thường xuyên sử dụng các máy chụp XQ kỹ thuật số, do vậy hình ảnh $X Q$ dễ lưu trữ tại cơ sở khám chữa bệnh, cũng như bệnh nhân cũng có thể lưu trữ trong các email cá nhân của mình để so sánh kết quả giữa các lần điều trị, hay dùng các hình ảnh tổn thương này là công cụ để dạy học, nghiên cứu, tư vấn... Nha khoa từ xa đang trở nên cấp thiết trong cộng đồng, đặc biệt trong cuộc cách mạng công nghệ khoa học kỹ thuật 4.0, thì việc ban đầu khảo sát các bệnh lý thường gặp ở địa phương, hướng dẫn thăm khám và sử dụng các biện pháp dự phòng bệnh răng miệng ở các vùng sâu, vùng xa trở nên dễ dàng hơn [1]. Trong đó, $X Q$ kỹ thuật số đóng một vai trò không nhỏ. Tuy nhiên, các dữ liệu ảnh mẫu của các bênh nhân chưa được tâp hợp và lưu trữ một cách có hệ thống tại các cơ sở điều trị. Phương pháp học máy có khả năng lưu trữ và cung cấp thông tin hồ sơ dữ liệu ảnh cũng như XQ cho bệnh nhân và bác sỹ. Vì vậy, sử dụng mố hình học máy để hố trợ chẩn đoán một số bệnh lý răng miệng thông thường, trong đó có bệnh lý răng khôn mọc lệch- một bệnh lý rất hay gặp có ý nghĩa rất lớn đối với cộng đồng trong việc dự phòng bệnh, giảm thiểu cũng như giảm chi phí điều trị các biến chứng kèm theo, cải thiện chức năng ăn nhai [2],[3],[4],[5]. Ngoài ra việc sử dụng điện thoại thông minh và mạng internet phủ rộng tại Việt Nam tạo điều kiện cho việc truyên hình ảnh đi xa với chất lượng đảm bảo, hỗ trợ được cho công tác tư vấn từ xa.

Trên thế giới, đã có nhiều nghiên cứu về ứng dụng của học máy trong việc hỗ trợ chẩn đoán một số bệnh lý răng miệng thông thường như sâu răng, viêm quanh cuống[6],[8],[9]... Ớ Việt Nam, học máy, trí tuệ nhân tạo cũng đã được nghiên cứu trong một số lĩnh vực như ung thư, sản khoa, nha khoa [7]. Chính vì thế, với mong muốn đánh giá liệu học máy có thể hỗ trợ cho bác sỹ, cộng đồng chẩn đoán và đưa ra lời khuyên nha khoa hay không, chúng tôi đã tiến hành nghiên cứu đề tài "Xây dứng hệ thống hỗ trợ chẩn đoán bệnh lý răng khồn mọc lệch bằng phương pháp học máy". 
II. ĐỐI TƯỢNG VÀ PHƯƠNG PHÁP NGHIÊN CỨU

Nghiên cứu được thực hiện tại Trung tâm khám chữa bệnh kỹ thuật cao Răng Hàm Mặt, Viện Đào tạo Răng Hàm Mặt, Trường ĐH Y Hà Nội; Bệnh viện Việt Nam - Cuba và một số địa điểm khác.

Nghiên cứu được thực hiện theo phương pháp thử nghiệm lẩm sàng để xác định giá trị chẩn đoán của một phương pháp.

Tiêu chuẩn chọn mẫu: Bệnh nhân có răng khôn mọc lệch Bệnh nhân đồng ý tham gia nghiên cứu.

Tiêu chuẩn loại trừ: Bệnh nhân tâm thần hoặc mất ổn định tâm lý, bệnh toàn thân chưa điều trị ổn định, hình ảnh, Ẋ-Quang không thể quan sát được tổn thương.

Cỡ mẫu. Tính cõ mẩu nghiên cứu sử dụng công thức để đánh giá độ nhạy và độ đặc hiệu của một phương pháp chẩn đoán bệnh:

$$
n_{s e}=\left(\frac{Z_{a s}^{2} \times p_{s e} \times\left(1-p_{s e}\right)}{w^{2}}\right) / p_{\text {dis }}
$$

Trong đó: $\mathrm{n}_{\text {se: }}$ cõ mẫu nghiên cứu cho độ nhạy, là hằng số của phân phối chuẩn (chọn $a=$ 0,05 thì bằng 1,96 ),

pse là xác xuất dương tính thật (độ nhạy) (ước tính là $80 \%$ ); w là sai số của hai xác xuất dương tính thật (độ nhạy) và âm tính thật (độ đặc hiệu), lấy $w=0,05$

- Bệnh răng khôn mọc lệch: lấypdis= 0,574 (theo nghiên cứu của Hashemipour MA và cộng sự, năm 2013, với N = 1020)

$$
n_{s e}=\left(\frac{1 s^{9}, 6^{2} \times 0,80 \times(1-0,80)}{0,05^{2}}\right) / 0,574=428
$$

Thực tế kho dữ liệu thu thâp được 504 trường hợp. Thử nghiệm độ nhạy và độ đặc hiệu được tiến hành trên 100 bệnh nhân.

Để thử nghiệm trước tên cần xây dựng kho dữ liệu, xây dựng phần mềm, kiến trúc hệ thống đề xuất và sau đó tiến hành thử nghiệm

\section{Phương pháp xây dựng bộ dữ liệu:}

Xây dựng bộ dữ liệu dựa trên các tiêu chuẩn chọn mấu, tiêu chuẩn loại trừ. Quá trình thu thập mẫu được thực hiên từ quá trình thăm khám trực tiếp của bác sỹ tại một số cơ sở như: Viện Đào tạo Răng Hàm Mặt - Trường Đại học $Y$ Hà Nội, Khoa Răng Miệng - Bệnh viện Việt Nam - Cu Ba và một số cơ sở khác.

\section{Các bước tiến hành nghiên cứu:}

- Thu thâp kho dữ liêu

- Xây dựng kho dữ liệu

- Xây dựng thuật toán hỗ trợ chẩn đoán bệnh răng khôn mọc lệch

- Cài đặt thuật toán hỗ trợ chẩn đoán răng khôn mọc lệch trên nền tảng Andriod và iOS.

- Tập huấn cho nhóm nghiên cứu sử dụng phần mềm hiểu và sử dụng được phần mềm trên các thiết bị thông minh.

- Triển khai thử nghiệm hiệu quả hỗ trợ chuẩn đoán của phần mềm học máy: Nghiên cứu này bao gồm ba giai đoạn:

+ Giai đoạn 1: Khám bệnh nhân trên lâm sàng

+ Giai đoạn 2: Khám qua phần mềm hỗ trợ chẩn đoán (bảng câu hỏi, XQ...) bởi một nhóm bác sỹ khác

+ Giai đoạn 3: Nhập liệu và xử lý số liệu bằng phần mềm SPSS16.0.

Nghiên cứu thực hiện tuân thủ theo các qui định của đạo đức nghiền cứu y sinh học, nghiên cứu đã được thông qua hội đồng đạo đức y sinh học của Trường Đại học Y Hà nội, mã số IRBVN1001.

Cách tính độ nhạy, độ đặc hiệu và độ chính xác: Độ nhạy của một xét nghiệm là tỷ lể những trường hợp thực sự có bệnh và có kết quả xét nghiệm dương tính trong toàn bộ các trường hợp có bệnh, và được tính dựa vào công thức:

\section{Số dương tính thật \\ Độ nhạy = $\frac{\text { Số dương tính thật }+ \text { Số âm tính giả }}{\text { ẩ }}$}

Độ đặc hiệu của một xét nghiệm là tỷ lệ những trường hợp thực sự không có bệnh và có kết quả xét nghiệm âm tính trong toàn bộ các trường hợp không bị bệnh. Độ đặc hiệu được tính theo công thức sau:

\section{Số âm tính thật \\ Độ đặc hiệu $=\frac{\text { Số âm tính thật }+ \text { Số dương tính giả }}{\text { Sộ }}$}

Độ chính xác của một xét nghiệm là tỷ lệ những trường hợp có kết quả xét nghiệm khớp với tình trạng có/không có bệnh trong toàn bộ các trường hợp được xét nghiệm. Độ chính xác được tính theo công thức sau:

$$
\text { Độ chính xác }=\frac{\text { Số âm tính thật + Số dương tính thật }}{\text { Toàn bộ các trường hợp được xét nghiệm }}
$$

\section{KẾT QUẢ NGHIÊN CỨU}

Kho dữ liệu được thu thập từ 504 phim XQ Panorama của 504 bệnh nhân có răng khôn mọc lệch. Vùng tổn thương răng khôn mọc lệch nhìn thấy được trên phim Panorama được đánh dấu lại như qui ước.Các ảnh có độ phân giải đảm bảo yêu cầu nghiên cứu. Trong đó độ phân giải của ảnh XQ Panorama thấp nhất là 2444 x 1292 . Kho dữ liệu được thực hiện trong đề tài được lưu trữ trên cấu trúc dữ liệu của chuẩn PASCAL_VOC. Định dạng PASCAL_ VOC là một trong hai định 
dạng phổ biến (cùng với định dạng COCO) trong việc xây dựng kho dữ liệu cho các bài toán nhận diện đối tượng, phù hợp với các thuật toán học máy, đặc biệt là các kỹ thuật học sâu (deep learning). Cấu trúc dữ liệu theo định dạng PASCAL_VOC được xây dựng dựa trên các tệp tin có định dạng XML. Kho dữ liệu của gồm 3 thư mục chính. Thư mục Annotations bao gồm các file XML chứa thông tin về các dữ liệu tổn thương đã được mã hóa. Thông tin về các tổn thương trong ảnh bao gồm vị trí tổn thương, kích thước vùng tổn thương theo khuôn dạng được quy định bởi định dạng Pascal_VOC.

\section{Hiêu quả chẩn đoán}

Nghiên cứu thử nghiệm tính độ nhạy và độ đặc hiệu được thực hiện trên 100 bệnh nhân với tổng số răng khổn hàm dưới là 187 răng trên phim Panorama. Trong tổng số 187 răng khôn hàm dưới, theo bác sỹ chẩn đoán 63 răng mọc thẳng, chiếm 33,7\%, 124 răng mọc lệch, chiếm $66,3 \%$. Theo phân loại, loại 1 có 42 răng $(22,5 \%)$, loại 2 có 81 răng $(43,3 \%)$ và loại 4 có 1 răng $(0,5 \%)$. Tuy nhiên, khi sử dụng phần mềm để chẩn đoán chúng tôi có kết quả như sau: máy chẩn đoán được 187 răng (100\%). Máy chẩn đoán giống bác sỹ ở 149 răng (79,68\%).

Bảng 1. Kêt quả chẩn đoán của máy (theo phân loại Parant).

\begin{tabular}{|c|c|c|c|c|c|c|c|}
\hline & \multicolumn{5}{|c|}{ Chẩn đoán của máy } & \multirow{2}{*}{ Tổng số } \\
\hline & & Loại 0 & Loai 1 & Loại 2 & Loai 3 & Loại 4 & \\
\hline \multirow{4}{*}{$\begin{array}{c}\text { Chẩn } \\
\text { đoán } \\
\text { của } \\
\text { bác sỹ }\end{array}$} & Loại 0 & $53(84,1 \%)$ & $8(12,7 \%)$ & $0(0 \%)$ & $0(0 \%)$ & $2(3,2 \%)$ & $63(100 \%)$ \\
\hline & Loại 1 & $9(21,4 \%)$ & $23(54,8 \%)$ & $10(23,8 \%)$ & $0(0 \%)$ & $0(0 \%)$ & $42(100 \%)$ \\
\hline & Loại 2 & $3(3,7 \%)$ & $3(3,7 \%)$ & $73(90,1 \%)$ & $0(0 \%)$ & $2(2,5 \%)$ & $81(100 \%)$ \\
\hline & Loại 4 & $1(100 \%)$ & $0(0 \%)$ & $0(0 \%)$ & $0(0 \%)$ & $0(0 \%)$ & $1(100 \%)$ \\
\hline
\end{tabular}

Độ nhạy và độ đặc hiệu của chẩn đoán bằng phần mềm hợc máy. Đối với việc xác định có hay không có răng khôn mọc lệch, độ nhạy của máy là $89,5 \%$ và độ đặc hiệu là $84,1 \%$. Nói cách khác, trong số các răng khôn được nghiên cứu, cứ 100 răng mọc lệch thì máy chẩn đoán đúng hơn 89 răng, $84,1 \%$ số răng mọc thẳng được máy chẩn đoán đúng.

Bảng 2. Độ nhạy, độ đặc hiệu và độ chính xác khỉ chẩn đoán răng khôn mọc lệch bằng học máy

\begin{tabular}{|c|c|c|}
\hline & $\begin{array}{c}\text { Máy } \\
\text { chẩn đoán }\end{array}$ & $\begin{array}{c}\text { Tiêu chuấn } \\
\text { vàng }\end{array}$ \\
\hline Dương tính & 121 & 124 \\
\hline Dương tính thật & 111 & \\
\hline Dương tính giả & 10 \\
\hline Độ nhạy & \multicolumn{2}{|c|}{$89,5 \%$} \\
\hline Ạm tính & 66 & 63 \\
\hline Ám tính thật & 53 & \\
\hline Âm tính giả & 13 \\
\hline Độ đặc hiệu & \multicolumn{2}{|c|}{$84,1 \%$} \\
\hline \multicolumn{3}{|c|}{ Độ chính xác } \\
\hline \multicolumn{2}{|c|}{ Máy chấn đoán giống bác sỹ } \\
\hline Loại 0 & \multicolumn{2}{|c|}{53} \\
\hline Loại 1 & \multicolumn{2}{|c|}{23} \\
\hline Loại 2 & \multicolumn{2}{|c|}{73} \\
\hline Loại 4 & \multicolumn{2}{|c|}{0} \\
\hline Độ chính xác & $79,68 \%$ \\
\hline
\end{tabular}

\section{BÀN LUẬN}

Đối với giai đoạn này, chúng tôi tập trung bàn luận về hiệu năng chẩn đoán của máy khi so với tiêu chuẩn vàng là chẩn đoán của bác sỹ. Từ đó đưa ra nhận xét về tính khả thi đối với việc áp dụng nghiên cứu này ra ngoài thực tế.

Độ chính xác (Accuracy) chung của chẩn đoán ở mức tốt, với giá trị 79,68\%. Trong đó, hai phân loại chiếm tỉ lệ cao nhất là phân loại 2 và 0 đều có độ chính xác trên $84 \%$. Phân loại 1 đứng sau với $54,8 \%$. Nếu lấy cutoff là răng khôn mọc lệch và không mọc lệch (phân loại 0: không lệch, 1-4: lệch), chúng tôi có được kết quả độ nhạy $89,5 \%$ và độ đặc hiệu $84,1 \%$. Kết quả này cho thây hiệu năng chẩn đoán của máy tính ở mức độ tốt khi nhận diện răng khôn mọc lệch. Đối với việc đánh giá từng phân loại, chúng tôi không dùng test độ nhạy và độ đặc hiệu vì nó không có ý nghĩa nhiều trên lâm sàng. Vì vậy, chúng tôi sử dụng test đánh giá độ tương hợp (Weighted Kappa và ICC) giữa chẩn đoán của máy và bác sỹ. Chỉ số Weighted Kappa cho giá trị 0,72 và ICC cho giá trị 0,7 . Cả hai chỉ số đều nằm trong khoảng từ $0,61-0,8$, tức là ở mức tốt, nếu trên 0,8 là mức rất tốt. Nói cách khác, chẩn đoán giữa máy và bác sỹ có độ tương đồng ở mức tốt, có thể áp dụng trên lâm sàng.

\section{KẾT LUẬN}

Khi sử dụng học máy để hỗ trợ chẩn đoán bệnh lý răng khôn mọc lệch: độ nhạy, độ đặc hiệu tương ứng lần lượt là $98,5 \%$; $86 \%$ khi chẩn đoán có hay không có bệnh lý. Để nâng cao chất lượng mô hình chẩn đoán sử dụng công nghệ học máy, nên đầu tư thêm nguồn lực để mở rộng kho dữ liệu Cần thu thập nhiều hớn nữa các thể bệnh khác nhau, đặc biệt là các thể bệnh ít 
gặp để học máy có thể chẩn đoán được tốt hơn. Số lượng dữ liệu càng nhiều và càng phong phú thì khả năng nhận diện được tổn thương càng tốt hơn. Cơ sở hạ tầng công nghệ thông tin (CNTT) cần được đầu tư tốt để đảm bảo hiệu năng làm việc của hệ thống.

\section{TÀI LIÊU THAM KHẢO}

1. Fernandez-Millan, R., Medina-Merodio, J. A., Plata, R. B., Martinez-Herraiz, J. J., \& Gutierrez-Martinez, J. M. (2015). A laboratory test expert system for clinical diagnosis support in primary health care. Applied Sciences, 5(3), 222-240.

2. Oliveira, J., \& Proença, H. (2011), Caries detection in panoramic dental X-ray images, Computational Vision and Medical Image Processing,Springer Netherlands, 175-190.

3. Duong DL, Kabir MH, Kuo RF. Automated caries detection with smartphone color photography using machine learning. Health Informatics Journal. 2021;27(2):14604582211007530.

4. Lee JH, Kim DH, Jeong SN. Diagnosis of cystic lesions using panoramic and cone beam computed tomographic images based on deep learning neural network. Oral Diseases. 2020. 26(1):152-158.

5. Berdouses ED, Koutsouri GD, Tripoliti EE, et al. A computer-aided automated methodology for the detection and classification of occlusal caries from photographic color images. 2015;62:119-135.

6. Srivastava MM, Kumar P, Pradhan L, Varadarajan SJapa. Detection of tooth caries in bitewing radiographs using deep learning. 2017.

7. Ngan, T. T., Tuan, T. M., Minh, N. H., \& Dey, N. (2016). Decision Making Based on Fuzzy Aggregation Operators for Medical Diagnosis from Dental X-ray images. Journal of medical systems, 40(12), 280, 1-7

8. Girshick R, Donahue J, Darrell T, Malik JJItopa, intelligence $\mathrm{m}$. Region-based convolutional networks for accurate object detection and segmentation. 2016;38(1):142-158.

9. Lee H, Park M, Kim J. Cephalometric landmark detection in dental $\mathrm{x}$-ray images using convolutional neural networks. Paper presented at: Medical Imaging 2017: Computer-Aided Diagnosis2017.

\section{BÁo CÁO SỰ Cố Y KHOA TẠI BỆNH VIỆN ĐA KHOA TỈNH PHÚ THỌ NĂM 2020}

\section{TÓM TẮT}

Báo cáo sự cố y khoa là một vấn đề mới đối với y tế Viêt Nam, minh chứng nổi bật nhất chính là số liêu về báo cáo sự cố y khoa của nước ta được công bố còn ít hơn rất nhiếu so với các nước khác và so với thực tế nó xảy ra. Mong muốn bước đầu đưa ra những số liệu có cơ sở khoa học về vấn đề báo cáo sự cố y khoa, giúp cho công tác cải tiến chất lượng bệnh viện đa khoa tỉnh Phú Thọ nói riêng và chất lượng dịch vụ y tế nói chung cho nước nhà. Nghiên cứu mố tả cắt ngang phân tích số liệu thứ cấp là toàn bộ các báo cáo sự cố y khoa của bệnh viện từ tháng 5 năm 2019 đến tháng 4 năm 2020. Có 365 sự cố y khoa đước NVYT của bệnh viện báo cáo. Trong đó: Điều dưỡng thực hiện báo cáo 62,7\%; Cán bộ thuộc chuyển mổn khối nôi báo cáo $52,6 \%$; Hình thức báo cáo tự nguyện 97,5\%; Một số thông tin ghi nhận trong báo cáo sự cố: mô tả ngắn gọn về sự cố $100 \%$, xử trí ban đầu $87,9 \%$, khoa điều trị của người bệnh $55,6 \%$, thông báo sự cố tới người bệnh 47,7\%; ghi nhận sự cố vào hồ sơ bệnh án 23,8\%, thông báo sự

\footnotetext{
${ }^{1}$ Trung tâm y tế huyện Tân Sơn tỉnh Phú Thọ ¿Sở y tế Phú Tho

${ }^{3}$ Trường đại học Y tế công công

${ }^{4}$ Bênh viện đa khoa tỉnh Phú Tho

Chịu trách nhiệm chính: Nguyễn Huy Ngọc

Email: huyngoc888@gmail.com

Ngày nhận bài: 11.3.2021

Ngày phản biên khoa hoc: 12.5.2021

Ngày duyệt bài: 18.5.2021
}

Kiều Quang Phát ${ }^{1}$, Nguyễn Huy Ngọc $^{2}$, Nguyễn Thị Kim Ngân ${ }^{3}$, Nguyễn Quang Ần ${ }^{4}$

cố tới người nhà $14,0 \%$, thông báo sự cố tới bác sỹ $7,1 \%$. Sự cố y khoa được báo cáo là vấn đề khó của bệnh viện, báo cáo tự nguyện đã được nhân viên y tế thực hiện, thông tin trong báo cáo đa phần còn chưa đầy đủ theo yêu cầu báo cáo.

Tư khóa: Báo cáo sự cố y khoa.

\section{SUMMARY}

\section{MEDICAL INCIDENT REPORTING AT PHU THO} GENARAL PROVINCIAL HOSPITAL 2020

Medical incident reports are a new issue for Vietnamese health, the most prominent proof is that the published data on medical incident reports in our country are much less than other countries, and it is different and than it actually happens. Desiring to initially give out scientifically based data on medical incident reporting, helping to improve the quality of Phu Tho province general hospital in particular and the quality of medical services in general for our country. The descriptive study cross-section analyzes the secondary data that are all hospital medical incident reports from May 2019 to April 2020. There are 365 medical incidents reported by the health worker of the hospital. In which: Nursing reports $62.7 \%$; Staff of internal expertise reports $52.6 \%$; Form of voluntary reporting $97.5 \%$; Some information recorded in the incident reports: brief description of the incident $100 \%$, initial management $87.9 \%$, patient's department $55.6 \%$, reporting the incident to the patient $47.7 \%$, record the incident in the medical record $23.8 \%$, report the incident to family members $14.0 \%$, report the incident to the doctor $7.1 \%$. The 